\title{
APPROXIMATE INNERNESS OF POSITIVE LINEAR MAPS OF FINITE VON NEUMANN ALGEBRAS
}

\author{
HIDEO TAKEMOTO
}

Dedicated to Professor Yoshinao Misonou on his 60th birthday

\begin{abstract}
Let $M$ be a $\sigma$-finite, finite von Neumann algebra with a faithful, normalized, normal trace $\operatorname{Tr}$ and a positive linear map $\rho$ of $M$ into itself. If $\rho$ is approximately inner with respect to the norm $\|\cdot\|_{2}$ induced by $\operatorname{Tr}$, then $\rho$ is closely related to a $*$-homomorphism. In particular, if $\rho$ is unital and approximately inner, then $\rho$ is a $*$-homomorphism of $M$ into itself.
\end{abstract}

1. Introduction. We examine the fact that positive linear maps with an approximate innerness are close to $*$-homomorphisms. Let $M$ be a factor of type $\mathrm{II}_{1}$, and let $\operatorname{Aut}(M)$ (resp. Int $(M))$ be the automorphism group (resp. inner automorphism group) of $M$. As a result of the $u$-topology (see Haagerup [2] and Sakai $[4]), \operatorname{Aut}(M)$ is a topological group. Now, since $M$ is a finite factor, the $u$-topology on $\operatorname{Aut}(M)$ coincides with the topology of strong pointwise convergence. Furthermore, by Sakai [4], if $M$ is a hyperfinite factor of type $\mathrm{II}_{1}$, the closure of $\operatorname{Int}(M)$ is $\operatorname{Aut}(M)$. Completely positive maps are closely related to automorphisms in the field of $C^{*}$-algebras and von Neumann algebras. Thus, we consider the approximate innerness of positive linear maps of von Neumann algebras. In this paper we deal with finite von Neumann algebras.

Throughout this paper let $M$ be a $\sigma$-finite von Neumann algebra with a faithful, normalized, normal trace Tr. We use a norm $\|\cdot\|_{2}$ on $M$ defined by $\|x\|_{2}=$ $\operatorname{Tr}\left(x^{*} x\right)^{1 / 2}$.

Let $A$ and $B$ be $C^{*}$-algebras. A linear map $\rho$ from $A$ to $B$ is said to be $n$-positive if the multiplicity map $\rho_{n}$ from the matrix algebra $M_{n}(A)$ over $A$ to the algebra $M_{n}(B)$ over $B$, defined by $\rho_{n}\left(\left[a_{i j}\right]\right)=\left[\rho\left(a_{i j}\right)\right]$, is positive. If $\rho$ is $n$-positive for every $n$, we call $\rho$ completely positive. Many authors (for example, $[\mathbf{1}, \mathbf{5}, \mathbf{6}$, Chapter IV, $\S 3,7])$ have studied the completely positive maps of $C^{*}$-algebras. In particular, we have Stinespring's theorem [5]: Let $A$ be a $C^{*}$-algebra and $\rho$ a completely positive map of $A$ to $B(H)$, where $B(H)$ is the von Neumann algebra of all bounded operators on a Hilbert space $H$. Then there exists a representation $\pi$ of $A$ to a Hilbert space $K$ and a bounded operator $v$ of $H$ to $K$ such that $\rho(x)=v^{*} \pi(x) v$ for every $x \in A$.

In particular, if $\rho$ is unital (i.e., $\rho(1)=1$ ), then $v$ is an isometry. Furthermore, if $A$ is a von Neumann algebra and $\rho$ is normal, then $\pi$ is a normal representation. In general, we cannot take the above operator $v$ in $A$. For this problem we have the following result by Haagerup [3, Proposition 2.1]: Let $N$ be a properly infinite von Neumann algebra, and let $F$ be a finite-dimensional subfactor. Let $\rho$ be a completely positive map from $F$ to $N$. Then there exists an element $a \in N$ such

Received by the editors August 12, 1984.

1980 Mathematics Subject Classification. Primary 46L10, 46L30, 46L40.

Key words and phrases. Approximate innerness, trace, completely positive map, finite von Neumann algebra. 
that $\rho(x)=a^{*} x a$ for every $x \in F$. In this paper we consider the problem for finite von Neumann algebras by using approximate innerness.

2. Results. In this section we show that a certain positive linear map of a finite von Neumann algebra $M$ into itself is closely related to a $*$-homomorphism of $M$ into itself. Before we present the main theorem we mention the following lemma by Choi $[\mathbf{1}]$ (see also [7]).

LEMMA 1. Let $A$ and $B$ be unital $C^{*}$-algebras, and let $\rho$ be a unital completely positive map from $A$ to $B$. If $\rho$ is a $C^{*}$-homomorphism (i.e., $\rho\left(a^{2}\right)=\rho(a)^{2}$ for every selfadjoint element a of $A$ ), then $\rho$ is a $*$-homomorphism of $A$ to $B$.

In this paper we consider the positive linear maps with approximate innerness. Thus, we introduce the notation of approximate innerness.

DEFINITION 2. Let $M$ be a $\sigma$-finite, finite von Neumann algebra with a fixed, faithful, normalized, normal trace Tr. A positive linear map $\rho$ from $M$ into $M$ is approximately inner if there exists a net $\left\{a_{\lambda}\right\}$ (not necessarily bounded) in $M$ satisfying $\lim \left\|\rho(x)-a_{\lambda}^{*} x a_{\lambda}\right\|_{2}=0$ for every $x \in M$.

Under the above definition, if $\rho$ is a positive linear map and approximately inner, then $\rho$ is a completely positive map from $M$ to $M$. To show this we must show by [6, Chapter IV, Corollary 3.4] that for every natural number $n$,

$$
\sum_{i, j=1}^{n} y_{i}^{*} \rho\left(x_{i}^{*} x_{j}\right) y_{j} \geq 0
$$

for every $x_{1}, \ldots, x_{n}$ and $y_{1}, \ldots, y_{n}$ in $M$. Since $\operatorname{Tr}$ is a faithful normal trace,

$$
\sum_{i, j=1}^{n} y_{i}^{*} \rho\left(x_{i}^{*} x_{j}\right) y_{j} \geq 0 \Leftrightarrow \operatorname{Tr}\left(\left(\sum_{i, j=1}^{n} y_{i}^{*} \rho\left(x_{i}^{*} x_{j}\right) y_{j}\right) h\right) \geq 0
$$

for every $h \geq 0$ in $M$. Now, since the map $x \rightarrow a_{\lambda}^{*} x a_{\lambda}$ on $M$ is completely positive for every $\lambda \in \Lambda$,

$$
\operatorname{Tr}\left(\left(\sum_{i, j=1}^{n} y_{i}^{*} a_{\lambda}^{*} x_{i}^{*} x_{j} a_{\lambda} y_{j}\right) h\right) \geq 0 .
$$

Furthermore, by the assumption of approximate innerness for $\rho$,

$$
\begin{aligned}
\lim & \left|\operatorname{Tr}\left(\left(\sum_{i, j=1}^{n} y_{i}^{*} a_{\lambda}^{*} x_{i}^{*} x_{j} a_{\lambda} y_{j}\right) h\right)-\operatorname{Tr}\left(\left(\sum_{i, j=1}^{n} y_{i}^{*} \rho\left(x_{i}^{*} x_{j}\right) y_{j}\right) h\right)\right| \\
& =\lim \left|\sum_{i, j=1}^{n} \operatorname{Tr}\left(\left(a_{\lambda}^{*} x_{i}^{*} x_{j} a_{\lambda}-\rho\left(x_{i}^{*} x_{j}\right)\right) y_{j} h y_{i}^{*}\right)\right|=0 .
\end{aligned}
$$

Thus, we can show that

$$
\operatorname{Tr}\left(\left(\sum_{i, j=1}^{n} y_{i}^{*} \rho\left(x_{i}^{*} x_{j}\right) y_{j}\right) h\right) \geq 0
$$

for every positive element $h$ in $M$, so $\sum_{i, j=1}^{n} y_{i}^{*} \rho\left(x_{i}^{*} x_{j}\right) y_{j} \geq 0$. Therefore, $\rho$ is a completely positive map from $M$ to $M$. 
Thus, we can show that such a positive map is closely related to a $*$-homomorphism.

THEOREM 3. Let $M$ be a $\sigma$-finite, finite von Neumann algebra. Let $\rho$ be a positive linear map from $M$ to $M$ and approximately inner with respect to a net $\left\{a_{\lambda}\right\}$ such that $\left\|a_{\lambda}^{*} a_{\lambda}-e\right\|_{2} \rightarrow 0$ and $\left\|a_{\lambda} a_{\lambda}^{*}-f\right\|_{2} \rightarrow 0$ for some projections $e$ and $f$ in $M$. Then $\rho$ is a *-homomorphism from the von Neumann algebra $f M f$ to the von Neumann algebra eMe.

ProOF. By the assumption for the net $\left\{a_{\lambda}\right\}$ and approximate innerness of $\rho$ with respect to $\left\{a_{\lambda}\right\}, \rho(1)=e$ and $\rho(1-f)=0$. Thus we can assume that $f a_{\lambda} e=a_{\lambda}$ for every $\lambda \in \Lambda$. By the remark following Definition 2, $\rho$ is a completely positive map. So $\rho$ is a unital, completely positive map from von Neumann algebra $f M f$ to von Neumann algebra $e M e$. To show that $\rho$ is a $*$-homomorphism from $f M f$ to $e M e$, we must show by Lemma 1 that $\rho\left(x^{2}\right)=\rho(x)^{2}$ for every selfadjoint element $x \in f M f$. Given an arbitrary selfadjoint element $x \in f M f$, then

$$
\left\|\rho(x)-a_{\lambda}^{*} x a_{\lambda}\right\|_{2}^{2}=\operatorname{tr}\left(\rho(x)^{2}\right)-2 \operatorname{Tr}\left(\rho(x) a_{\lambda}^{*} x a_{\lambda}\right)+\operatorname{Tr}\left(a_{\lambda}^{*} x a_{\lambda} a_{\lambda}^{*} x a_{\lambda}\right) .
$$

Now, since

$$
\begin{aligned}
\left|\operatorname{Tr}\left(a_{\lambda}^{*} x a_{\lambda} a_{\lambda}^{*} x a_{\lambda}-a_{\lambda}^{*} x^{2} a_{\lambda}\right)\right| & =\left|\operatorname{Tr}\left(a_{\lambda}^{*} x\left(a_{\lambda} a_{\lambda}^{*}-f\right) x a_{\lambda}\right)\right| \\
& =\left|\operatorname{Tr}\left(\left(a_{\lambda} a_{\lambda}^{*}-f\right) x a_{\lambda} a_{\lambda}^{*} x\right)\right| \\
& \leq \operatorname{Tr}\left(\left(a_{\lambda} a_{\lambda}^{*}-f\right)^{2}\right)^{1 / 2} \operatorname{Tr}\left(x a_{\lambda} a_{\lambda}^{*} x^{2} a_{\lambda} a_{\lambda}^{*} x\right)^{1 / 2} \\
& \leq\left\|a_{\lambda} a_{\lambda}^{*}-f\right\|_{2} \cdot\|x\| \operatorname{Tr}\left(x a_{\lambda} a_{\lambda}^{*} a_{\lambda} a_{\lambda}^{*} x\right)^{1 / 2} \\
& \leq\|x\|^{2} \cdot\left\|a_{\lambda} a_{\lambda}^{*}-f\right\|_{2} \cdot \operatorname{Tr}\left(a_{\lambda} a_{\lambda}^{*} a_{\lambda} a_{\lambda}^{*}\right)^{1 / 2} \\
& =\|x\|^{2} \cdot\left\|a_{\lambda} a_{\lambda}^{*}\right\|_{2} \cdot\left\|a_{\lambda} a_{\lambda}^{*}-f\right\|_{2},
\end{aligned}
$$

$\left\{\left\|a_{\lambda} a_{\lambda}^{*}\right\|_{2}\right\}$ is bounded, and $\lim \left\|a_{\lambda} a_{\lambda}^{*}-f\right\|_{2}=0$, we have

$$
\lim \left\{\operatorname{Tr}\left(a_{\lambda}^{*} x a_{\lambda} a_{\lambda}^{*} x a_{\lambda}\right)-\operatorname{Tr}\left(a_{\lambda}^{*} x^{2} a_{\lambda}\right)\right\}=0 .
$$

Thus, since $\lim \operatorname{Tr}\left(a_{\lambda}^{*} x^{2} a_{\lambda}\right)=\operatorname{Tr}\left(\rho\left(x^{2}\right)\right)$ by the assumption,

$$
\lim \operatorname{Tr}\left(a_{\lambda}^{*} x a_{\lambda} a_{\lambda}^{*} x a_{\lambda}\right)=\operatorname{Tr}\left(\rho\left(x^{2}\right)\right) .
$$

Furthermore, since

$$
\begin{aligned}
\left|\operatorname{Tr}\left(\rho(x) a_{\lambda}^{*} x a_{\lambda}\right)-\operatorname{Tr}\left(\rho(x)^{2}\right)\right| & =\left|\operatorname{Tr}\left(\rho(x)\left(a_{\lambda}^{*} x a_{\lambda}-\rho(x)\right)\right)\right| \\
& \leq\|\rho(x)\|_{2} \cdot\left\|\rho(x)-a_{\lambda}^{*} x a_{\lambda}\right\|_{2},
\end{aligned}
$$

$\lim \operatorname{Tr}\left(\rho(x) a_{\lambda}^{*} x a_{\lambda}\right)=\operatorname{Tr}\left(\rho(x)^{2}\right)$. By the above considerations and

$$
\lim \left\|\rho(x)-a_{\lambda}^{*} x a_{\lambda}\right\|_{2}=0
$$

we get

$$
\operatorname{Tr}\left(\rho(x)^{2}\right)-2 \operatorname{Tr}\left(\rho(x)^{2}\right)+\operatorname{Tr}\left(\rho\left(x^{2}\right)\right)=0 .
$$

So, $\operatorname{Tr}\left(\rho\left(x^{2}\right)-\rho(x)^{2}\right)=0$. Now, since $\rho$ is a completely positive map, $\rho(x)^{2} \leq \rho\left(x^{2}\right)$. Therefore, $\rho\left(x^{2}\right)=\rho(x)^{2}$, so, by Lemma $1, \rho$ is a $*$-homomorphism from $f M f$ to $e M E$.

REMARK 4. We assumed in Theorem 3 that $\left\|a_{\lambda}^{*} a_{\lambda}-e\right\|_{2} \rightarrow 0$ and $\left\|a_{\lambda} a_{\lambda}^{*}-f\right\|_{2}$ $\rightarrow 0$. If $\rho$ is approximately inner and $\rho(1)=e$ is a projection, then the above 
condition is equivalent to $\rho(1-f)=0$ and $\operatorname{Tr}(f)=\operatorname{Tr}(e)$. In fact, we have $\rho(1)=e$ and $\rho(1-f)=0$ for both cases. Thus, we can assume that $f a_{\lambda} e=a_{\lambda}$ for every $\lambda \in \Lambda$. Then from the relation

$$
\begin{aligned}
\left\|a_{\lambda}^{*} a_{\lambda}-e\right\|_{2}^{2} & =\operatorname{Tr}\left(\left(a_{\lambda}^{*} a_{\lambda}-e\right)^{2}\right)=\operatorname{Tr}\left(\left(a_{\lambda} a_{\lambda}^{*}-f\right)^{2}+e-f\right) \\
& =\left\|a_{\lambda} a_{\lambda}^{*}-f\right\|_{2}^{2}+\operatorname{Tr}(e-f)
\end{aligned}
$$

we can give the proof that the above conditions are equivalent.

From the last relation, if $\rho(1)=e$ is a projection and $\rho(1-f)=0$ for a projection $f$ satisfying $\operatorname{Tr}(f)<\operatorname{Tr}(e)$, then $\rho$ is not approximately inner.

COROllary 5. Let $\rho$ be a unital, positive, linear map of a $\sigma$-finite, finite von Neumann algebra to itself. If $\rho$ is approximately inner, then $\rho$ is a*-homomorphism.

Proof. Since $\rho(1)=1$, the relation $\lim \left\|\rho(x)-a_{\lambda}^{*} x a_{\lambda}\right\|_{2}=0$ for every $x \in M$ induces $\left\|a_{\lambda}^{*} a_{\lambda}-1\right\|_{2}=\left\|a_{\lambda} a_{\lambda}^{*}-1\right\|_{2} \rightarrow 0$. Thus, $\rho$ is a $*$-homomorphism by Theorem 3.

AKNOWLEDGEMENT. The author would like to thank the members of the seminar in Sendai, especially Professors Y. Misonou, S. Sakai, J. Tomiyama, and A. Kishimoto, for useful discussions.

\section{REFERENCES}

1. M. D. Choi, A Schwartz inequality for positive linear maps on $C^{*}$-algebras, Illinois J. Math. 18 (1974), 565-574.

2. U. Haagerup, The standard form of von Neumann algebras, Math. Scand. 37 (1975), 271-283.

3. _ A new proof of the equivalence of injectivity and hyperfiniteness for factors on a separable Hilbert space (preprint).

4. S. Sakai, On automorphism groups of $\mathrm{II}_{1}$-factors, Tôhoku Math. J. 26 (1974), 423-430.

5. W. F. Stinespring, Positive maps on $C^{*}$-algebras, Proc. Amer. Math. Soc. 6 (1955), 211-216.

6. M. Takesaki, Theory of operator algebras. I, Springer-Verlag, Berlin and New York, 1979.

7. J. Tomiyama, Complete positivity in operator algebras, Lecture Note No. 4, RIMS, Kyoto Univ., 1978. (Japanese)

Department of Mathematics, College of General Education, Tôhoku UniVERSITY, SENDAI 980, JAPAN 